\title{
Deriving the Beam Equation using the Minimum Total Potential Energy Principle and Solving the Equation Numerically
}

\author{
Magnus Komperød ${ }^{1}$ \\ ${ }^{1}$ Technological Analyses Centre, Nexans Norway AS, Norway, magnus. komperod@nexans.com
}

\begin{abstract}
The beam equation describes the deflection of a beam subject to point loads and / or distributed loads, while being supported at both ends. The beam equation is commonly derived in the scientific literature using force- and moment balances, which lead to a boundary value problem. The present paper derives the beam equation using the minimum total potential energy principle and solves the optimization problem numerically. The motivation behind this work is to ease future extensions of the beam equation into larger deflections and nonlinear materials. These future extensions are necessary to model subsea power cables and umbilicals during bending stiffness tests which is the author's final goal.

Keywords: Beam Equation, Bending Stiffness Test, Minimum Total Potential Energy Principle, Numerical Analysis, Subsea Power Cable, Umbilical.
\end{abstract}

\section{Introduction}

The offshore oil and gas industry, as well as power grid companies, require increasingly accurate analyses and physical testing of subsea power cables and umbilicals to be able to install them in deeper waters, in colder environments, and during harsher weather conditions. This challenges engineers, scientists, and software developers working in the cable manufacturing industry and among service- and software providers.

Subsea power cables and umbilicals are complex structures consisting of a large number of individual elements, such as the umbilical shown in Figure 1. For some cable designs the number of individual elements exceeds 200. The relative displacements between these elements and complex nonlinear material characteristics are among the main challenges in modeling cables' mechanical properties. As both the cable geometry and the material properties are highly complex, there will inherently be uncertainties in the cable models. It is therefore essential to validate the models through physical testing.

The scientific literature on physical bending stiffness tests of cables is very sparse. Hence, there is very limited information on various test rig designs; Maioli (2015) and Tarnowski (2015) both present results based on rigs with a design as sketched in Figure 2. In Maioli (2015) the arrangement is horizontal, i.e. the force is applied in the horizontal plane, while in Tarnowski (2015) the force

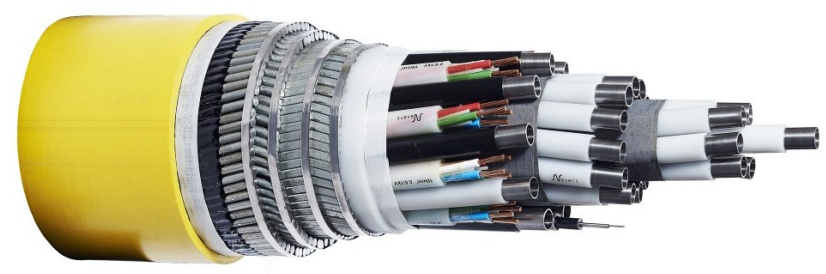

Figure 1. Umbilical with steel tubes, electric- and fiber optic signal cables, and armor wires.

is applied vertically.

The author and his colleagues have developed a bending stiffness rig which is also based on the principle illustrated in Figure 2. This work is presented in Jordal et al. (2017) and Komperød et al. (2017). The rig is vertical and the force can be applied in both directions, i.e. up and down, in a sinusoidal manner, making the cable oscillate.

In Komperød et al. (2017) the beam equation is used to calculate the cable's bending stiffness based on measurements of the cable's deflection and the force required to achieve this deflection. This equation is derived for beams under the assumptions of small deflections and linear, elastic material in the beam. The exact same equation is also used in Maioli (2015). Tarnowski (2015) presents another model that intends to handle larger deformations. However, also this approach considers the cable as one solid object, rather than a compound object consisting of a large number of individual cable elements.

When cables are bent, there are relative displacements between the cable elements. A large number of scientific publications consider the effect of shear forces between cable elements due to friction, for example Lutchansky (1969) and Kebadze (2000), and show that this effect has major impact on the cables' bending stiffness. Over the last few years, also shear forces due to bitumen-coating on the armor wires have got attention in the scientific literature. Among the publications on this topic are Hedlund (2015), Komperød (2016a,b), and Martindale et al. (2017).

Analyses of cables' mechanical properties are commonly (i) assuming constant cable bending curvature 

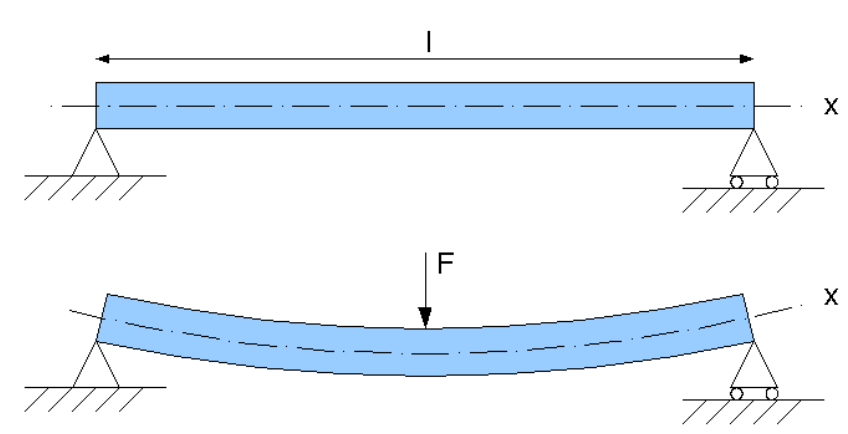

Figure 2. Sketch of bending stiffness rig. Illustration from www.wikimedia.org.

along the cable's length direction and (ii) neglecting the boundary conditions at the cable ends. However, to the author's knowledge, it is not possible to perform a physical bending stiffness test of a cable in such a way that the cable's physical boundary conditions will not influence the test results. Also, the condition of constant bending curvature is challenging to meet in real-life test rigs. Hence, the bending stiffness calculated in analyses and the bending stiffness identified from physical tests are not directly comparable because they are based on different assumptions.

To close the gap between analyses and physical testing, the author develops models of cables subject to the loads and the boundary conditions of the bending stiffness rig. These models can then be directly compared to, and hence validated against, the results of the physical tests. When the models have been validated, changing their loads and boundary conditions allows the same models to be used in the case of constant curvature and no boundary conditions as discussed above. This way the author intends to bridge the differences between analyses and physical testing which are currently preventing the analyses from being verified against physical testing in a consistent way. Due to the high complexity of the overall problem, the work will be split into 4-6 milestones.

This paper presents the results of the first milestone, which is to derive the beam equation using the minimum total potential energy principle and to solve this equation numerically. Using the minimum total potential energy principle, rather than force- and moment balances, is believed to simplify future extensions of the beam equation into large deflections, nonlinear materials, and correct modeling of shear forces between the cable elements. It is further believed that it will be impossible to reach an analytical solution to the overall problem. It is therefore desirable to develop a numerical solution during the first milestone which can later be extended when working towards future milestones.

\section{Nomenclature}

Table 1 presents the main nomenclature used in this paper. Variables without a specific physical meaning are defined in the main text where they first appear. Vectors are denoted with lower case, bold font. Matrices are denoted with upper case, bold font. Square bracket are used to denote parts of a vector or a matrix. For example $\mathbf{A}[1: 4,3]$ means the first four rows of the third column of A. Exponent notation applied to vectors, for example $\mathbf{x}^{2}$, means element-by-element exponent.

Table 1. Nomenclature.

\begin{tabular}{|c|c|}
\hline$\overline{\mathbf{c}}$ & $\begin{array}{l}\text { Vector with Clenshaw-Curtis quadrature } \\
\text { weights. }\end{array}$ \\
\hline $\mathbf{D}_{1}$ & Chebyshev first-derivative matrix. \\
\hline $\mathbf{D}_{2}$ & Chebyshev second-derivative matrix. \\
\hline EI & Bending stiffness $\left[\mathrm{Nm} /\left(\mathrm{m}^{-1}\right)\right]$ \\
\hline$g$ & Acceleration of gravity $\left[\mathrm{m} / \mathrm{s}^{2}\right]$. \\
\hline$L$ & Length between beam supports [m]. \\
\hline$M$ & Bending moment $[\mathrm{Nm}]$ \\
\hline$\tilde{m}$ & Mass per unit length $[\mathrm{kg} / \mathrm{m}]$ \\
\hline$N$ & Number of discretization points [-]. \\
\hline $\bar{N}$ & $\begin{array}{l}\text { Number of discretization points over a } \\
\text { half beam [-]. }\end{array}$ \\
\hline$P$ & Total potential energy $[\mathrm{J}]$ \\
\hline$P_{\mathrm{g}}$ & Gravitational energy $[\mathrm{J}]$. \\
\hline$P_{\mathrm{s}}$ & Strain energy $[\mathrm{J}]$ \\
\hline$s$ & Beam length parameter $[\mathrm{m}]$. \\
\hline$u$ & Vertical deflection of beam [m]. \\
\hline$u_{\mathrm{p}}$ & Vertical deflection of piston [m]. \\
\hline $\mathbf{u}$ & Vector containing discrete points of $u$. \\
\hline$x$ & Coordinate along $x$-axis $[\mathrm{m}]$ \\
\hline$\kappa$ & Bending curvature $\left[\mathrm{m}^{-1}\right]$. \\
\hline$\lambda_{i}$ & Lagrange multiplier no. $i$. \\
\hline
\end{tabular}

Figure 3 shows the Cartesian coordinate system used in this paper.

\section{Assumptions and Simplifications}

The mathematical derivation in this paper is subject to the following assumptions and simplifications:

1. The beam is subject to infinitesimal deflections only.

2. The beam is made of a linear, elastic material and has identical cross section over its entire length.

3. Only the beam length between the supports shown in Figure 2 is considered. That is, possible beam length outside these supports is disregarded. 


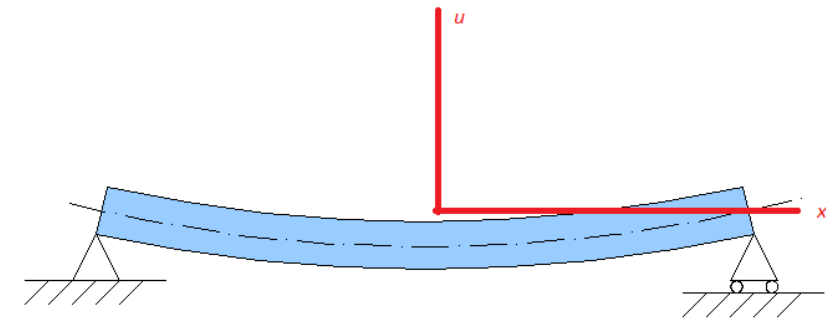

Figure 3. The Cartesian coordinate system used in this paper. Based on illustration from www.wikimedia.org.

4. The beam is assumed not to move horizontally at the center of its length, i.e. where the $F$ arrow points in Figure 2.

5. The developed model is quasi-static, i.e. inertia and kinetic energy are disregarded.

6. The beam's height is much smaller than the length between the supports, $L$.

\section{Mathematical Model}

This section derives the mathematical model of the total potential energy of the beam, as well as the constraints that follow from the physical test rig and the beam's physical properties. How to formulate the model and the constraints numerically, and how to numerically solve for the minimum total potential energy are derived in Section 5.

\subsection{Strain Energy in the Beam}

The bending stiffness of the beam, EI, is by definition

$$
\mathrm{EI} \stackrel{\text { def }}{=} \frac{M}{\kappa} .
$$

Solving Eq. 1 for the moment, $M$, and integrating w.r.t. the curvature, $\kappa$, gives the beam's strain energy, $P_{\mathrm{s}}$, per unit length, i.e.

$$
\begin{aligned}
\frac{\partial P_{\mathrm{s}}}{\partial s} & =\int_{0}^{\kappa} M \mathrm{~d} \kappa \\
& =\frac{1}{2} \mathrm{EI} \kappa^{2} .
\end{aligned}
$$

Hence, the total strain energy in the beam is

$$
\begin{aligned}
P_{\mathrm{S}} & =\int_{\text {beam length }} \frac{1}{2} \mathrm{EI} \kappa^{2} \mathrm{~d} s \\
& =\frac{1}{2} \mathrm{EI} \int_{\text {beam length }} \kappa^{2} \mathrm{~d} s,
\end{aligned}
$$

where $\kappa=\kappa(s)$. The integration limits will be discussed in more detail in Section 5.

\subsection{Gravitational Energy}

When choosing $u=0$ as the reference level for calculating the gravitational energy, $P_{\mathrm{g}}$, the gravitational energy per unit length is

$$
\frac{\partial P_{\mathrm{g}}}{\partial s}=\tilde{m} g u
$$

Hence, the gravitational energy over the entire beam is

$$
\begin{aligned}
P_{\mathrm{g}} & =\int_{\text {beam length }} \tilde{m} g u \mathrm{~d} s \\
& =\tilde{m} g \int_{\text {beam length }} u \mathrm{~d} s,
\end{aligned}
$$

where $u=u(s)$.

\subsection{Total Potential Energy}

The beam's total potential energy, $P$, is the sum of the strain energy and the gravitational energy, i.e.

$$
\begin{aligned}
P= & P_{\mathrm{S}}+P_{\mathrm{g}} \\
= & \frac{1}{2} \mathrm{EI} \int_{\text {beam length }} \kappa^{2} \mathrm{~d} s \\
& +\tilde{m} g \int_{\text {beam length }} u \mathrm{~d} s .
\end{aligned}
$$

\subsection{Constraints}

The purpose of finding the total potential energy in Eq. 6 is to minimize this expression w.r.t. the beam's deflection $u(s)$ over its length, $s$, which will give its total potential energy and its deflection profile. However, the beam is not completely free to move and deform; it is constrained by the test rig and by its own physical properties. In mathematical terms this translates into constraints that apply when minimizing Eq. 6 w.r.t. $u(s)$.

The bending stiffness rig developed by the author and his colleagues is as sketched in Figure 2. As the rig is

(3) designed to oscillate between positive and negative values of $u$ (i.e. up and down), the supports are made to both prevent the beam from being pushed down and from being lifted up. Similarly, the force $F$ is generated by a piston which can both push the beam down and lift it up. Hence, the constraints are 


$$
\begin{gathered}
u(\text { left end })=0, \\
u(\text { center })=u_{\mathrm{p}}, \\
u(\text { right end })=0,
\end{gathered}
$$

where $u_{\mathrm{p}}$ is the piston's position.

From beam theory it follows that the function $u(s)$ and its first- and second derivatives are continuous. This is also a constraint which can mathematically be expressed as

$$
u(s) \in C^{2},
$$

where $C^{2}$ is the set all functions which are continuous and have continuous first- and second derivatives (and possibly continuous higher order derivatives). The mathematical importance of the latter constraint will be further explained in Section 5.

\section{Numerical Solution}

Section 4 derives a mathematical model of the total potential energy in the beam, as well as the associated constraints. The present section solves the constrained optimization problem, i.e. finds the minimum total potential energy, using numerical mathematics.

\subsection{Implications of Assumptions and Simplifi- cations}

Assumptions 1 of Section 3 implies that the beam's length parameter $s$ will be identical to the $x$ coordinate. Assumptions 1 and 4 combined mean that the beam will not move horizontally at any point. Hence, the beam's length exactly spans the interval $\left[-\frac{L}{2}, \frac{L}{2}\right]$. It is then convenient to replace $s$ by $x$, and consider the beam over $x \in\left[-\frac{L}{2}, \frac{L}{2}\right]$. It then follows that the integration limits of Eqs. 3, 5, and 6 are $-\frac{L}{2}$ and $\frac{L}{2}$.

From the mathematical literature, the curvature of a graph $u(x)$ is known to be

$$
\kappa=\frac{\frac{\mathrm{d}^{2} u}{\mathrm{~d} x^{2}}}{\left(1+\left(\frac{\mathrm{d} u}{\mathrm{~d} x}\right)^{2}\right)^{\frac{3}{2}}}
$$

Under assumption $1, \frac{\mathrm{d} u}{\mathrm{~d} x}$ is very small compared to unity. Eq. 11 can then be approximated by

$$
\kappa=\frac{\mathrm{d}^{2} u}{\mathrm{~d} x^{2}}
$$

\subsection{Calculating the Strain Energy}

The overall purpose of the numerical solution of the beam equation is to find the $u(s)$ that minimizes the total potential energy subject to the constraints. To the author's knowledge, numerical methods are not able to find the exact function $u(s)$. What these methods instead do is (i) to find $u(s)$ at discrete points which allows subsequent interpolation, as well as numerical differentiation and quadrature (numerical integration), or (ii) to find the coefficients of a polynomial or a series, for example a Fourier series or a Chebyshev series, which then serves as an approximation to $u(s)$. In this paper it has been chosen to base the calculations on discrete points that are Chebyshevdistributed along $x$, because this distribution gives excellent convergence properties both in interpolation, differentiation, and quadrature.

The constraint of Eq. 10 requires $u(s)$ to be continuous and to have continuous first- and second derivatives. However, $u(s)$ does not have continuous third derivative at the point where the piston, i.e. the force $F$, pushes or pulls the beam. This is because the force gives an abrupt change in the beam's shear force. To prevent the abrupt third derivative from disturbing the excellent convergence properties of Chebyshev series for smooth functions, the function $u(s)$ is split in two halves; one half to the left of the force $F$, i.e. in the interval $\left[-\frac{L}{2}, 0\right\rangle$, and one half to the right of the force, i.e. the interval $\left\langle 0, \frac{L}{2}\right]$. Eq. 10 then imposes constraints on how the two halves should be connected.

The numbers of Chebyshev nodes over the left half and the right half are the same, namely $\bar{N}$. Then the total number of nodes is

$$
N=2 \bar{N}-1,
$$

because the center node is common for both halves. The nodes are organized in a column vector $\mathbf{u} \in \mathbb{R}^{N}$.

The following text explains how to calculate the strain energy in the left half of the beam as function of the discrete-point deflections of the vector $\mathbf{u}$. Let $\overline{\mathbf{u}}$ be the first $\bar{N}$ nodes of $\mathbf{u}$, i.e.

$$
\overline{\mathbf{u}} \stackrel{\text { def }}{=} \mathbf{u}[1: \bar{N}] \in \mathbb{R}^{\bar{N}} .
$$

Let $\overline{\mathbf{k}}$ be the vector with curvature values, $\kappa$, at the Chebyshev nodes. The curvature can be approximated by the second derivative as stated in Eq. 12. Hence, the curvatures can be calculated as

$$
\overline{\mathbf{k}}=\mathbf{D}_{2} \overline{\mathbf{u}}
$$

where $\mathbf{D}_{2}$ is the Chebyshev second-derivative matrix of dimension $\bar{N} \times \bar{N}$. The structure of this matrix is derived in Reid (2014). 
From Eq. 3 and the reasoning of Section 5.1, the strain energy in the left half of the beam can be approximated by

$$
P_{\mathrm{S}}(\text { left half })=\frac{1}{2} \mathrm{EI} \int_{-L / 2}^{0} \kappa^{2} \mathrm{~d} x .
$$

Eq. 15 gives that the strain energy per unit length at the Chebyshev nodes is $\frac{1}{2} \operatorname{EI}\left(\mathbf{D}_{2} \overline{\mathbf{u}}\right)^{2}$, where the superscript means element-by-element exponent. The strain energy in the left half of the beam is then given by Clenshaw-Curtis quadrature, i.e.

$$
P_{\mathrm{S}}(\text { left half })=\left(\frac{L}{4} \overline{\mathbf{c}}\right)^{T}\left(\frac{1}{2} \mathrm{EI}\left(\mathbf{D}_{2} \overline{\mathbf{u}}\right)^{2}\right),
$$

where $\overline{\mathbf{c}}$ is the vector containing the Chenshaw-Curtis quadrature weights for the standard quadrature interval $[-1,1]$. In the first parenthesis of Eq. 17, a factor $\frac{L}{2}$ is the quadrature interval width, while a factor $\frac{1}{2}$ is to cancel the width 2 of the standard interval. Clenshaw-Curtis quadrature is explained in Reid (2014).

Eq. 17 can be rewritten to

$$
P_{\mathrm{S}}(\text { left half })=\frac{\mathrm{EI} L}{8} \overline{\mathbf{u}}^{T} \mathbf{D}_{2}{ }^{T} \operatorname{diag}(\overline{\mathbf{c}}) \mathbf{D}_{2} \overline{\mathbf{u}},
$$

where the operator $\operatorname{diag}(\cdot)$ returns a diagonal matrix with the argument vector's elements on the diagonal. It is now convenient to define the symmetric matrix $\overline{\mathbf{W}}$ as

$$
\overline{\mathbf{W}} \stackrel{\text { def }}{=} \frac{\operatorname{EI} L}{4} \mathbf{D}_{2}^{T} \operatorname{diag}(\overline{\mathbf{c}}) \mathbf{D}_{2} \in \mathbb{R}^{\bar{N} \times \bar{N}} .
$$

Eq. 18 can then be written as

$$
P_{\mathrm{S}}(\text { left half })=\frac{1}{2} \overline{\mathbf{u}}^{T} \overline{\mathbf{W}} \overline{\mathbf{u}} .
$$

Eq. 20 gives an expression for the strain energy in the left half of the beam which is easy to handle, and in particular easy to differentiate. Differentiation is important for later finding the minimum. However, it is not straight forward how to extend the equation to also include the right half of the beam, while keeping it easy to differentiate, because the center node is included in the calculations of both halves. The following presents a simple solution. A block matrix $\mathbf{W}_{\mathrm{L}}$ is defined as

$$
\mathbf{W}_{\mathbf{L}} \stackrel{\text { def }}{=}\left[\begin{array}{cc}
\overline{\mathbf{W}} & \mathbf{0}_{\bar{N} \times(\bar{N}-1)} \\
\mathbf{0}_{(\bar{N}-1) \times \bar{N}} & \mathbf{0}_{(\bar{N}-1) \times(\bar{N}-1)}
\end{array}\right],
$$

where the subscript $\mathrm{L}$ means left, and $\mathbf{0}$ means the zero matrix of the dimension indicated by the subscripts. The strain energy in the left half of the beam can then be written as

$$
P_{\mathrm{S}}(\text { left half })=\frac{1}{2} \mathbf{u}^{T} \mathbf{W}_{\mathrm{L}} \mathbf{u} .
$$

Please note that $\mathbf{u}$, rather than $\overline{\mathbf{u}}$, is used in Eq. 22.

Making a similar reasoning as Eqs. 14-22 for the strain energy of the right half of the beam gives

$$
P_{\mathrm{S}}(\text { right half })=\frac{1}{2} \mathbf{u}^{T} \mathbf{W}_{\mathrm{R}} \mathbf{u},
$$

where

$$
\mathbf{W}_{\mathbf{R}} \stackrel{\text { def }}{=}\left[\begin{array}{cc}
\mathbf{0}_{(\bar{N}-1) \times(\bar{N}-1)} & \mathbf{0}_{(\bar{N}-1) \times \bar{N}} \\
\mathbf{0}_{\bar{N} \times(\bar{N}-1)} & \overline{\mathbf{W}}
\end{array}\right] .
$$

In Eq. 24 the subscript $R$ means right, and $\overline{\mathbf{W}}$ is the matrix defined in Eq. 19.

The strain energy over the entire beam is found by adding the two halves, i.e.

$$
\begin{aligned}
P_{\mathrm{S}} & =\frac{1}{2} \mathbf{u}^{T} \mathbf{W}_{\mathrm{L}} \mathbf{u}+\frac{1}{2} \mathbf{u}^{T} \mathbf{W}_{\mathrm{R}} \mathbf{u} \\
& =\frac{1}{2} \mathbf{u}^{T}\left(\mathbf{W}_{\mathrm{L}}+\mathbf{W}_{\mathrm{R}}\right) \mathbf{u} \\
& =\frac{1}{2} \mathbf{u}^{T} \mathbf{W} \mathbf{u},
\end{aligned}
$$

where

$$
\mathbf{W} \stackrel{\text { def }}{=} \mathbf{W}_{\mathrm{L}}+\mathbf{W}_{\mathrm{R}}
$$

Eq. 25 gives an expression for the strain energy in the entire beam which is easy to differentiate w.r.t. $\mathbf{u}$.

\subsection{Calculating the Gravitational Energy}

Numerical calculation of the gravitational energy resembles numerical calculation of the strain energy, but it is simpler, partly because it depends on $u(s)$ rather than its second derivative, and partly because it is linear rather than quadratic.

Using Eq. 5 and Clenshaw-Curtis quadrature, the gravitational energy of the left half of the beam can be approximated by

$$
P_{\mathrm{g}}(\text { left half })=\left(\frac{L}{4} \overline{\mathbf{c}}\right)^{T}(\tilde{m} g \overline{\mathbf{u}}),
$$

where $\overline{\mathbf{u}}$ is as defined in Eq. 14, and $\overline{\mathbf{c}}$ is the vector which elements are the Clenshaw-Curtis quadrature weights. Eq. 27 can be rewritten to 


$$
P_{\mathrm{g}}(\text { left half })=\mathbf{q}_{\mathrm{L}}^{T} \mathbf{u},
$$

for the vector $\mathbf{q}_{\mathrm{L}}$ defined as

$$
\mathbf{q}_{L} \stackrel{\text { def }}{=} \frac{\tilde{m} g L}{4}\left[\begin{array}{c}
\overline{\mathbf{c}} \\
\mathbf{0}_{(\bar{N}-1) \times 1}
\end{array}\right] .
$$

Similarly, the gravitational energy of the right half of the beam can be expressed as

$$
P_{\mathrm{g}}(\text { right half })=\mathbf{q}_{\mathrm{R}}^{T} \mathbf{u}
$$

for

$$
\mathbf{q}_{\mathrm{R}} \stackrel{\text { def }}{=} \frac{\tilde{m} g L}{4}\left[\begin{array}{c}
\mathbf{0}_{(\bar{N}-1) \times 1} \\
\overline{\mathbf{c}}
\end{array}\right] .
$$

The gravitational energy over the entire beam is then given by adding Eq. 28 and Eq. 30, i.e.

$$
\begin{aligned}
P_{\mathrm{g}} & =\mathbf{q}_{\mathrm{L}}^{T} \mathbf{u}+\mathbf{q}_{\mathrm{R}}^{T} \mathbf{u} \\
& =\left(\mathbf{q}_{\mathrm{L}}+\mathbf{q}_{\mathrm{R}}\right)^{T} \mathbf{u} \\
& =\mathbf{q}^{T} \mathbf{u},
\end{aligned}
$$

where

$$
\mathbf{q} \stackrel{\text { def }}{=} \mathbf{q}_{\mathrm{L}}+\mathbf{q}_{\mathrm{R}} \text {. }
$$

\subsection{Calculating the Total Potential Energy}

The total potential energy is calculated by adding Eq. 25 and Eq. 32. Hence the total potential energy is

$$
P=\frac{1}{2} \mathbf{u}^{T} \mathbf{W} \mathbf{u}+\mathbf{q}^{T} \mathbf{u} .
$$

\subsection{Constraints}

The constraints of Eqs. 7-9 are straight forward to convert to the vector notation used in the numerical calculation. The constraints become

$$
\begin{gathered}
\mathbf{u}[1]=0, \\
\mathbf{u}[\bar{N}]=u_{\mathrm{p}}, \\
\mathbf{u}[N]=0 .
\end{gathered}
$$

The constraint of Eq. 10 must be handled carefully: In the open intervals $\left(-\frac{L}{2}, 0\right)$ and $\left(0, \frac{L}{2}\right), u(x)$ is described by the polynomials which interpolates $\{u[0], \ldots, u[\bar{N}]\}$ and $\{u[\bar{N}], \ldots, u[N]\}$, respectively. Please recall that the corresponding $x$-values are Chebyshev-distributed, which means that the interpolating polynomials are guaranteed to converge to the true $u(x)$ as $\bar{N}$ and $N-\bar{N}$ increase. As $u(x)$ is described by polynomials in these open intervals, it meets $C^{\infty}$, and hence also $C^{2}$, in the intervals.

At the left endpoint, $u\left(-\frac{L}{2}\right)$ and its right-sided limit will be equal, because the limit is given by a polynomial which interpolates through the point $\left(-\frac{L}{2}, u\left(-\frac{L}{2}\right)\right)$. Hence, $u(x)$ is at least $C^{0}$ at the left endpoint. As $u(x)$ is not defined for $x<-\frac{L}{2}$, derivatives of any order only exist on the right side of $x=-\frac{L}{2}$, where they are given by the polynomial interpolation. Hence, $u(x)$ is $C^{\infty}$ at the left endpoint. A similar reasoning applies for the right endpoint as well, which concludes that also this endpoint is $C^{\infty}$.

At $x=0$, two polynomials that both interpolates through the point $(0, u(0))$ meet. Hence, $u(0)$ and the limits from both sides will be equal, which means that $u(x)$ is $C^{0}$ at $x=0$. However, there is no inherent effect that forces the two interpolating polynomials to have the same derivatives at $x=0$. This is true for derivatives of any orders. Therefore, for $u(x)$ to be $C^{2}$ at $x=0$, equal first- and second derivatives at both sides of $x=0$ must be explicitly enforced as constraints, i.e.

$$
\lim _{x \rightarrow 0^{-}} \frac{\partial u}{\partial x}=\lim _{x \rightarrow 0^{+}} \frac{\partial u}{\partial x}
$$

$$
\lim _{x \rightarrow 0^{-}} \frac{\partial^{2} u}{\partial x^{2}}=\lim _{x \rightarrow 0^{+}} \frac{\partial^{2} u}{\partial x^{2}} .
$$

In terms of the discrete-point representation of $u(x)$, Eq. 38 can be approximated by

$$
\mathbf{D}_{1}[\bar{N},:] \mathbf{u}[1: \bar{N}]=\mathbf{D}_{1}[1,:] \mathbf{u}[\bar{N}: N],
$$

where $\mathbf{D}_{1}$ is the Chebyshev first-derivative matrix of dimension $\bar{N} \times \bar{N}$. Eq. 40 can be written as

$$
\mathbf{p}^{T} \mathbf{u}=0
$$

where the vector $\mathbf{p}$ is defined by

$$
\begin{aligned}
& \mathbf{p}_{\mathrm{L}} \stackrel{\text { def }}{=}\left[\mathbf{D}_{1}[\bar{N},:] \quad \mathbf{0}_{1 \times(\bar{N}-1)}\right]^{T},
\end{aligned}
$$

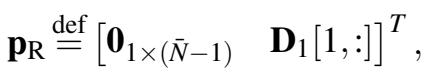

$$
\begin{aligned}
& \mathbf{p} \stackrel{\text { def }}{=} \mathbf{p}_{\mathrm{L}}-\mathbf{p}_{\mathrm{R}} \text {. }
\end{aligned}
$$

A similar reasoning for the second derivatives gives 
Further, the matrix $\mathbf{A}$ of Eq. 50 is the block matrix

$$
\mathbf{r}^{T} \mathbf{u}=0
$$

where

$$
\begin{aligned}
& \mathbf{r}_{\mathrm{L}} \stackrel{\text { def }}{=}\left[\mathbf{D}_{2}[\bar{N},:] \quad \mathbf{0}_{1 \times(\bar{N}-1)}\right]^{T},
\end{aligned}
$$

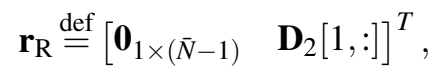

$$
\begin{aligned}
& \mathbf{r} \stackrel{\text { def }}{=} \mathbf{r}_{L}-\mathbf{r}_{R} \text {. }
\end{aligned}
$$

\subsection{Minimization subject to Constraints}

The total potential energy given by Eq. 34 should be minimized w.r.t. $\mathbf{u}$ subject to the constraints given in Eqs. 35, $36,37,41$, and 45 . An intuitive approach is to use the five constraints to eliminate five elements from the vector $\mathbf{u}$, and then minimize for the remaining $N-5$ elements of $\mathbf{u}$. This is indeed a decent and feasible approach. However, the elimination process will disturb the simple formulation of Eq. 34 and complicate the solution process somewhat. From the author's point of view, implementing the constraints using Lagrange multipliers is a simpler and more elegant approach that does not obscure the formulation of Eq. 34. Also, the Lagrange multipliers are themselves interesting variables, many with physical interpretations.

The Lagrange function of the constrained optimization problem is

$$
\begin{aligned}
L\left(\mathbf{u}, \lambda_{1}, \lambda_{2}, \lambda_{3}, \lambda_{4}, \lambda_{5}\right)= & \frac{1}{2} \mathbf{u}^{T} \mathbf{W} \mathbf{u}+\mathbf{q}^{T} \mathbf{u} \\
& -\lambda_{1} \mathbf{u}[1] \\
& -\lambda_{2}\left(\mathbf{u}[\bar{N}]-u_{\mathrm{p}}\right) \\
& -\lambda_{3} \mathbf{u}[N] \\
& -\lambda_{4} \mathbf{p}^{T} \mathbf{u} \\
& -\lambda_{5} \mathbf{r}^{T} \mathbf{u} .
\end{aligned}
$$

The expression of Eq. 49 is quadratic. Hence, it can be written on the form

$$
L(\mathbf{v})=\frac{1}{2} \mathbf{v}^{T} \mathbf{A} \mathbf{v}+\mathbf{b}^{T} \mathbf{v},
$$

where the vector $\mathbf{v}$ is the vector $\mathbf{u}$ augmented with the five Lagrange multipliers, i.e.

$$
\mathbf{v} \stackrel{\text { def }}{=}\left[\begin{array}{c}
\mathbf{u} \\
\lambda_{1} \\
\lambda_{2} \\
\lambda_{3} \\
\lambda_{4} \\
\lambda_{5}
\end{array}\right] \in \mathbb{R}^{N+5}
$$

$$
\begin{aligned}
& \mathbf{A} \stackrel{\text { def }}{=}\left[\begin{array}{cccccc}
\mathbf{W} & -\mathbf{i}_{1} & -\mathbf{i}_{\bar{N}} & -\mathbf{i}_{N} & -\mathbf{p} & -\mathbf{r} \\
-\mathbf{i}_{1}{ }^{T} & 0 & 0 & 0 & 0 & 0 \\
-\mathbf{i}_{\bar{N}}{ }^{T} & 0 & 0 & 0 & 0 & 0 \\
-\mathbf{i}_{N}{ }^{T} & 0 & 0 & 0 & 0 & 0 \\
-\mathbf{p}^{T} & 0 & 0 & 0 & 0 & 0 \\
-\mathbf{r}^{T} & 0 & 0 & 0 & 0 & 0
\end{array}\right] \\
& \in \mathbb{R}^{[(N+5) \times(N+5)]},
\end{aligned}
$$

where $\mathbf{i}_{j}$ is the $j$ th column of the $N \times N$ identity matrix. The vector $\mathbf{b}$ of Eq. 50 is

$$
\mathbf{b} \stackrel{\text { def }}{=}\left[\begin{array}{c}
\mathbf{q} \\
0 \\
u_{\mathrm{p}} \\
0 \\
0 \\
0
\end{array}\right] \in \mathbb{R}^{N+5} .
$$

The solution of the constrained optimization problem is the $\mathbf{v}$ vector that gives a stationary point to the Lagrange function. The derivative of the Lagrange function is

$$
\frac{\mathrm{d} L}{\mathrm{~d} \mathbf{v}}=\mathbf{A v}+\mathbf{b} .
$$

Hence, the solution of the constrained optimization problem is the solution of the linear system with $N+5$ equations and $N+5$ unknowns

$$
\mathbf{A v}=-\mathbf{b} .
$$

\section{Results and Interpretations}

The numerical calculations derived in this paper, which are based on the minimum total potential energy principle, have been compared to the formulas based on forceand moment balances. The two approaches give identical results for $\bar{N} \geq 5$, while they differ for lower values of $\bar{N}$. This conclusion is to be expected because the forceand-moment-balances approach gives $u(x)$ as a forth order polynomial, and it requires five interpolation points from the numerical calculations to give a forth order polynomial.

Figure 4 presents a comparison of the two approaches for a steel tube with bending stiffness $124.1 \mathrm{kNm}^{2}$ and specific mass $8.257 \mathrm{~kg} / \mathrm{m}$. The length between the supports is $3.000 \mathrm{~m}$ and the vertical deflection of the piston is $u_{\mathrm{p}}=-3.200 \times 10^{-2} \mathrm{~m}$. These input values are from a steel tube tested in the physical bending stiffness rig. The physical test is presented in Jordal et al. (2017).

The first $N$ elements of the $\mathbf{v}$ vector found from Eq. 55 is $\mathbf{u}$, i.e. the nodes shown in Figure 4. The latter five 


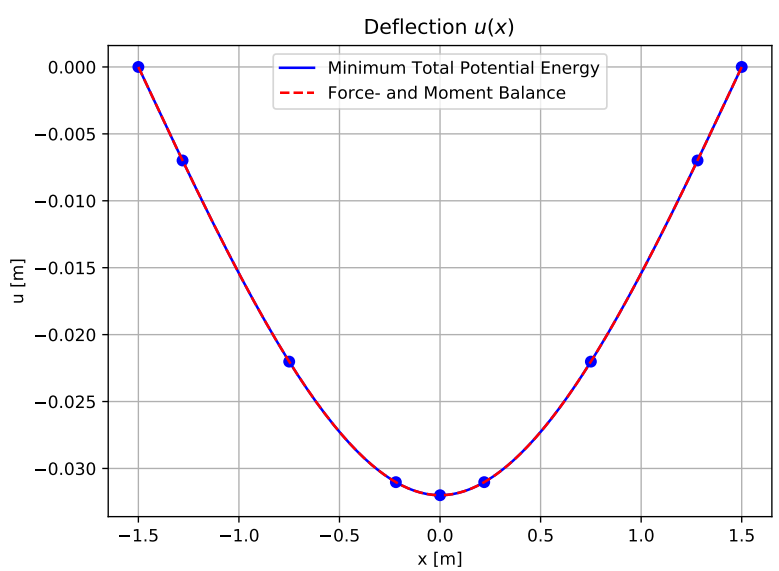

Figure 4. Comparison of $u(s)$ found from (i) the minimum total potential energy principle as derived in this paper, and (ii) from the approach based on force- and moment balances. The blue dots represent the values calculated by the former method, and the blue, solid line represents the polynomial interpolations between these points. $\bar{N}=5$ is used in the example.

elements of $\mathbf{v}$ are the five Lagrange multipliers defined in Eq. 49. The physical interpretation of each Lagrange multiplier is the derivative of the total potential energy, $P$, w.r.t. the constraint associated with the multiplier. Hence, $\lambda_{1}$ and $\lambda_{3}$ are the forces from the left and right beam supports, respectively, toward the beam, as shown in Figure 2. Similarly, $\lambda_{2}$ is the force from the piston toward the beam, i.e. the force $F$ in the figure. Multiplier $\lambda_{4}$ can be shown to be the beam's bending moment at $x=0$.

The last multiplier, $\lambda_{5}$, expresses the total potential energy's sensitivity to discontinuities in the bending curvature at $x=0$. To the author's knowledge, this formulation does not correspond to a well-known physical variable. $\lambda_{5}$ is a factor $10^{16}$ smaller than the other multipliers, measured in absolute value. Hence, $\lambda_{5}$ is assumed to be zero in the case of infinite arithmetic precision, which means that the corresponding constraint would have been met even if it was not enforced.

\section{Conclusions and Further Work}

The present paper derives numerical calculation of the beam equation based on the minimum total potential energy principle under the assumptions of linear material and small deflections. The calculation gives identical results as the derivation based on force- and moment balances which is commonly presented in the literature.

The work presented in this paper is the first milestone towards the author's final goal of modeling subsea power cables and umbilicals during bending stiffness tests. The future milestones are to include the effects of large deflections, nonlinear materials, and shear forces between the cable elements.

It is believed that the minimum total potential energy principle is more suitable for the future extensions than force- and moment balances. It is further believed that it will be impossible to reach an analytical solution of the overall problem due to its complexity, which is the reason for using numerical mathematics.

\section{References}

J. Hedlund. Modelling of viscoelastic dynamic bending stiffness for VIV analysis of submarine cables. In Proceedings of the 9th International Conference on Insulated Power Cables (Jicable '15), 2015.

L. Jordal, R. Slora, E. Vermeer, and M. Komperød. A novel bending stiffness rig for identification of subsea cables' and umbilicals' sensitivity to temperature under sinusoidal curvature oscillations. In Proceedings of the Twenty-seventh International Ocean and Polar Engineering Conference (ISOPE 2017), San Francisco, California, USA, 2017.

E. Kebadze. Theoretical modelling of unbonded flexible pipe cross-sections. PhD thesis, South Bank University, 2000.

M. Komperød. A frequency-domain model of bitumen-coated armor wires in subsea power cables, umbilicals, and power umbilicals. In Proceedings of the 35th International Conference on Ocean, Offshore and Arctic Engineering (OMAE 2016), Busan, South Korea, 2016a.

M. Komperød. Analytical calculation of capacity for bitumencoated armor wires in subsea cables and umbilicals. In Proceedings of the Twenty-sixth International Ocean and Polar Engineering Conference (ISOPE 2016), Rhodes, Greece, 2016 b.

M. Komperød, J. I. Juvik, G. Evenset, R. Slora, and L. Jordal. Large-scale tests for identifying the nonlinear, temperaturesensitive, and frequency-sensitive bending stiffness of the NordLink cable. In Proceedings of the 36th International Conference on Ocean, Offshore and Arctic Engineering (OMAE 2017), Trondheim, Norway, 2017.

M. Lutchansky. Axial stress in armor wires of bent submarine cables. Journal of Engineering Industry, 91(3):687 - 693, 1969.

P. Maioli. Bending stiffness of submarine cables. In Proceedings of the 9th International Conference on Insulated Power Cables (Jicable '15), 2015.

H. Martindale, S. Rossiter, T. Sheldrake, and R. Langdon. Improved dynamic structural modelling for subsea power cables with bitumen coated armour wires. In Proceedings of the 36th International Conference on Ocean, Offshore and Arctic Engineering (OMAE 2017), Trondheim, Norway, 2017.

H. Reid. Massachusetts Institute of Technology - Lecture notes 18.330 Introduction to numerical analysis, 2014.

J. Tarnowski. Improved method of determining bending stiffness of underground cables. In Proceedings of the 9th International Conference on Insulated Power Cables (Jicable '15), 2015. 